\title{
LEVANTAMENTO DO PERFIL SOCIAL, DEMOGRÁFICO E ECONÔMICO DE PACIENTES ATENDIDOS NA CLÍNICA DE ODONTOLOGIA DO CENTRO UNIVERSITÁRIO DE ARARAQUARA - UNIARA
}

DOMINGOS, Patricia Aleixo dos Santos. Professora Doutora da disciplina de Odontologia em Saúde

Coletiva do Centro Universitário de Araraquara - UNIARA. Av. Dr. Rocha Lima, 572, Jardim Adalgisa, 14.806-146, Araraquara, São Paulo, Brasil. E-mail: patyaleixo01 @ yahoo.com.br.

ROSSATO, Emersom Mocheti; BELLINI, Afonso. Cirurgiões-dentistas formados pelo Centro Universitário de Araraquara - UNIARA.

\begin{abstract}
Resumo
O conhecimento do perfil social, demográfico e econômico dos pacientes que recorrem aos serviços de saúde em uma Instituição de Ensino Superior - IES permite um planejamento de ações de educação e prevenção em saúde bucal das principais doenças que acometem esses indivíduos, assim como uma melhora no atendimento clínico de excelência prestado para a população que necessita de cuidados odontológicos. Com esse objetivo, o presente trabalho propôs avaliar o perfil social, demográfico e econômico dos pacientes que recorreram ao serviço odontológico oferecido pela Clínica do Curso de Graduação em Odontologia do Centro Universitário de Araraquara - UNIARA. Para isso, a amostra foi composta por conveniência e formada por indivíduos maiores de 13 anos, que procuraram atendimento ou que já estavam em tratamento no período de agosto a novembro de 2011 e março a junho de 2012, totalizando 198 indivíduos. Foi utilizado um questionário précodificado, com perguntas sobre os aspectos sociais e demográficos e também dados econômicos, de acordo com os Critérios de Classificação Econômica Brasil (CCEB 2011-base LSE 2009). Com base na metodologia utilizada, e os dados obtidos tabulados e expressos em números e porcentagens, conclui-se que a maior parte dos indivíduos é formada pelo gênero feminino $(65,66 \%)$, pertencente à classe $\mathrm{C}(57,57 \%)$ e procurou atendimento odontológico pelo motivo de dor (35,86\%). Dessa forma, este estudo resultou em dados relevantes que proporcionarão maior atenção à qualidade de planejamento dos atendimentos clínicos oferecidos à população que anualmente recorre ao Curso de Odontologia da Instituição de Ensino Superior estudada.
\end{abstract}

Palavras-chave: Odontologia em saúde pública; Serviços de saúde; Fatores socioeconômicos.

A SURVEY OF THE SOCIAL, DEMOgRAPHIC AND ECONOMICAL PROFILE OF PATIENTS ATTENDED aT THE OdONTOLOGIC Clinic of Centro Universitário de Araraquara - Uniara

\section{Abstract}

The knowledge of the social, economic and demographic features of patients who use health services in a Higher Education Institution - HEI allows the planning of education and prevention in oral health of major diseases that affect these individuals, as well as enhanced excellence in clinical care offered to the population in need of dental care. With this aim, this paper evaluated the social, demographic and economic profile of patients who used the dental services offered by the Clinic of Undergraduate Dentistry of Centro Universitário de Araraquara - UNIARA. For this purpose, the sample was formed by convenience and consisted of individuals older than thirteen years who sought treatment or who were already receiving treatment in the period from August to November 2011 and from March to June 2012, totaling 198 individuals. It was used a pre-coded questionnaire with questions on social and demographic as well as economic data according to the criteria of Brazil Economic Classification (CCEB 2011-based LSE 2009). Based on the chosen methodology and on the 
obtained data, tabulated and expressed in numbers and percentages, it is possible to conclude that the majority of individuals is formed by females $(65.66 \%)$ belonging to the $\mathrm{C}$ class $(57.57 \%)$ and sought dental care because of pain (35.86\%). Thus, this study resulted in relevant data that will provide greater attention to the planning quality of the clinical care offered to the population that annually makes use of the School of Dentistry, Higher Education Institution studied.

Keywords: Public Health Dentistry; Health Services; Socioeconomic Factors.

\section{INTRODUÇÃO}

O ensino da Odontologia na formação dos novos profissionais deve permitir que estes compreendam a realidade vivida pela população e sejam cidadãos que norteiam seu trabalho de acordo com as questões sociais do país, e não somente com a preocupação em sua formação técnica, na concorrência de mercado e visando ao retorno financeiro. No passado, o ensino apresentava-se exclusivamente tecnicista e voltado apenas para mercado de trabalho, o que não atendia aos interesses da comunidade, uma vez que ele não contribuía para a melhoria da realidade social brasileira.

Nesse contexto, mesmo que ainda as Universidades apresentem estrutura curricular com disciplinas estritamente teóricas, o ensino nesta área do conhecimento está fortemente baseado em atividades práticas realizadas pelos alunos nas clínicas das faculdades. Nesse ambiente, os futuros profissionais da saúde aprendem, exercem ações, assimilam condutas e adquirem hábitos, podendo fazer da Instituição de Ensino um espaço privilegiado de aprendizado dos procedimentos técnicos e, principalmente, da reflexão ética sobre sua responsabilidade social.

As unidades de saúde municipais prestam, em sua maioria, serviços odontológicos de atenção básica, que não suprem integralmente as necessidades da população. Dessa forma, as Universidades têm reforçado o seu papel de prestadora de serviços odontológicos à sociedade.
Brandini et al. (2008) também verificaram que as Universidades funcionam como prestadoras de serviços à comunidade e, por isso, as clínicas odontológicas pertencentes às IES devem atender às demandas acadêmicas e dos usuários que as procuram e, assim, estarem preparadas para resolução dos problemas da população.

Além disso, também é importante coletar informações consistentes sobre a situação socioeconômica dos pacientes para um correto levantamento de suas necessidades, a fim de aprimorar o atendimento da população. Tal fato estimulará a participação da comunidade no acesso aos serviços de saúde.

De acordo com pesquisa realizada por Baldani et al. (2004), há evidências da correlação entre os indicadores de desenvolvimento social relacionados à renda, moradia e escolaridade com cárie dentária, sendo o fator renda insuficiente diretamente associado às piores condições de saúde bucal encontradas. Outro relato na literatura mostrou a estreita relação entre os padrões sociais e a situação bucal dos indivíduos, mesmo nos países onde há declínio da prevalência da doença cárie.

Portanto, conhecer como cada indivíduo percebe a própria saúde é um importante passo para se compreender o padrão de procura por serviços prestados na área. Os resultados da Pesquisa Nacional por Amostras de Domicílios - PNAD de 1998 mostraram que o principal motivo de não procurar um atendimento de saúde foi a ausência de necessidade percebida (96\%). Além disso, outro motivo pela falta de iniciativa em recorrer aos atendimentos em saúde seria a dificuldade financeira, a qual foi apontada em segundo lugar na pesquisa $(1,3 \%)$.

É importante ressaltar que a população brasileira sofreu grandes alterações sociais e econômicas nas últimas décadas, o que pode ter representado mudanças no perfil de procura pelos serviços em saúde, sejam eles públicos ou privados. Apesar de existirem vários estudos sobre o perfil dos pacientes com necessidades especiais atendidos em Universidades, a literatura, no que diz respeito aos dados dos 
atendimentos clínicos prestados pelas IES à população em geral, mostra-se escassa e desatualizada. Por esse motivo, há a necessidade de se levantar tais dados na região de Araraquara/SP, vez que esta cidade pode ser considerada, ainda nos dias atuais, uma referência em ensino, pesquisa e extensão na área da Odontologia. O conhecimento sobre os dados sociais, demográficos e econômicos da população facilitaria o entendimento dos principais tipos de procedimentos clínicos realizados na Universidade, assim como o planejamento logístico e de recursos materiais e humanos para oferecer excelência na prestação de serviços à comunidade local.

O atendimento odontológico prestado pela Clínica de Odontologia da UNIARA é de baixo custo e realizado principalmente por alunos da graduação. Pesquisa realizada em 2010 pelo serviço de assistência social dessa unidade mostrou que 1.546 indivíduos foram triados no serviço da graduação e emergência, e 1.020 se tornaram pacientes atendidos na Clínica. Entretanto, não há um levantamento que defina o perfil dos pacientes que recorrem a esta instituição, como já foi encontrado em 1984 na Faculdade de Odontologia de Ribeirão Preto - USP, onde o paciente é definido como "dona de casa, com idade média de 35 anos, membro de família com 4 a 6 integrantes, casa própria e média salarial de 3 salários mínimos para o sustento. Outra pesquisa sobre o perfil dos pacientes foi realizada por Abreu e Oliveira, em 2002, quando constataram que a predominância é por pacientes do gênero feminino, domésticas/do lar e estudantes, sendo os procedimentos mais procurados os de diagnóstico e controle das doenças cárie e periodontal.

Diante do exposto, o estudo justifica-se pela necessidade de maior atenção à qualidade de atendimento da população de Araraquara e região, no que se refere aos serviços de saúde bucal que alcancem os princípios de promoção, proteção e recuperação do paciente. Assim, conhecer o perfil social, demográfico e econômico dos pacientes atendidos na Clínica de Odontologia da UNIARA possibilitará o planejamento das disciplinas práticas direcionado às reais necessidades do público que a procuram.

\section{Proposição}

O estudo avaliou o perfil social, biodemográfico e econômico dos pacientes atendidos na Clínica de Odontologia do Centro Universitário de Araraquara UNIARA.

\section{MATERial e métodos}

A realização deste estudo esteve vinculada à aprovação do Comitê de Ética em Pesquisa do Centro Universitário de Araraquara - UNIARA sob protocolo $\mathrm{n}^{\circ} 1277 / 11$.

\section{Caracterização da amostra}

O estudo foi do tipo transversal, também denominado de estudo de prevalência, que consiste em observar eventos e fatores em estudo ao mesmo tempo. Verificou-se que o serviço odontológico da UNIARA atendia em média 1.077 pessoas num intervalo de um (1) ano, das quais 686 eram adolescentes, adultos e idosos; os outros 391 pacientes eram crianças menores de 13 anos e portadores de necessidades especiais. Para obter o número de amostragem que representava a margem de erro de $6 \%$ e nível de confiança de $95,5 \%$, a casuística foi calculada baseada na fórmula estatística para populações finitas, que resultou em uma amostra de 198 participantes.

A amostra foi então delineada por conveniência e composta por 198 indivíduos, de ambos os gêneros, com faixa etária acima de 13 anos de idade, que recorreram ao atendimento na Clínica de Odontologia da UNIARA nos períodos de agosto a novembro de 2011 e março a junho de 2012.

Para definir os critérios de composição da amostra, foram consideradas as condições de atendimento dos pacientes nas diferentes disciplinas clínicas oferecidas no Curso de Graduação em Odontologia na UNIARA. Somente as especialidades de Odontologia Preventiva, Odontopediatria e Pacientes Especiais não foram incluídas na amostra por possuírem pacientes abaixo dos 13 anos de idade. Assim, todos os adolescentes e adultos que recorreram ao atendimento nas demais disciplinas foram entrevistados quando aceitaram 
participar como voluntários na pesquisa.

A seleção dos entrevistados ocorreu de forma aleatória, à medida que estes compareciam na Clínica de Odontologia da UNIARA. Destaca-se, ainda, que não houve perda amostral.

É importante salientar que foi adotado como critério de inclusão do voluntário na amostra o adequado Preenchimento e assinatura do Termo de Consentimento Pré-Informado.

\section{Instrumento de avaliação}

Como instrumento da pesquisa utilizou-se um formulário com 16 questões fechadas e 2 abertas, aplicado por meio de entrevista dirigida. Os assuntos abordados na coleta de dados referem-se a: condições sociais, demográficas, econômicas e de necessidade de tratamento. Parte do questionário é composta pelo Critério de Classificação Econômica Brasil (CCEB 2011 base LES 2009), adotado pela Associação brasileira de empresas de pesquisa para a classificação econômica da população brasileira. O CCEB é um instrumento de segmentação econômica que utiliza o levantamento de critérios domiciliares para diferenciar a população. $\mathrm{O}$ critério atribui pontos em função de cada característica domiciliar e realiza a soma destes pontos. É feita então uma correspondência entra faixas de pontuação do critério e extratos de classificação econômica definidos por A1, A2, B1, B2, C1, C2, D, E.

\section{Aspectos Sociais e Biodemográficos}

Os participantes da pesquisa foram inicialmente questionados sobre suas condições sociodemográficas, a saber: gênero, idade, estado civil, perfil social (escolaridade, número de moradores na residência), profissão e município/bairro de origem.

\section{Aspectos econômicos}

As questões referentes às condições econômicas abrangiam os seguintes temas: renda familiar, tipo de moradia (aluguel, casa própria, casa cedida) e localização (urbana ou rural), propriedade de bens duráveis (automóvel, geladeira, aparelho de microondas e televisor) e meio de transporte (individual ou coletivo).

\section{Necessidade de tratamento}

O motivo pelo qual procurou a Clínica; queixa principal para atendimento (dor, incômodo, rotina, baixo custo, entre outros).

O referido instrumento de avaliação foi aplicado uma única vez na forma de entrevista estruturada realizada pelos pesquisadores para auxiliar na compreensão das questões e no preenchimento das possíveis alternativas. É importante salientar que o entrevistador, em hipótese alguma, deveria influenciar o participante da pesquisa quando o mesmo não soubesse responder a qualquer uma das questões aplicadas, devendo a pergunta permanecer sem resposta.

\section{Análise estatística}

Os indicadores coletados foram inseridos em planilha eletrônica para procedimentos das análises descritivas, tendo sido utilizado o programa Excel 2010. Para descrever a amostra, foram utilizadas tabelas e gráficos contendo frequências absolutas e relativas.

\section{Resultados}

Os resultados da pesquisa elaborada com os pacientes atendidos na Clínica de Odontologia da UNIARA, no período de agosto a novembro de 2011 e março a junho de 2012, foram distribuídos em três diferentes tabelas, de acordo com os aspectos sociais e biodemográficos (Tabela 1), aspectos econômicos (Tabela 2) e necessidade de tratamento (Tabela 3) e um gráfico referente ao nível de escolaridade (Gráfico 1).

A tabela 1 apresenta a frequência de respostas obtidas sobre os aspectos sociais e biodemográficos dos participantes da pesquisa. 
Tabela 1 - Distribuição de frequência absoluta (n) e relativa (\%) dos aspectos sociais e biodemográficos dos pacientes que buscaram atendimentos na Clínica de Odontologia da UNIARA - Araraquara, 20112012.

\begin{tabular}{|c|c|c|c|}
\hline Perguntas & Respostas & $\begin{array}{c}\text { Número de } \\
\text { Pessoas }\end{array}$ & Porcentagem \\
\hline \multirow[t]{2}{*}{ Gênero: } & Masculino & 68 & $34,34 \%$ \\
\hline & Feminino & 130 & $65,66 \%$ \\
\hline \multirow{7}{*}{ Idade: } & $13-20$ & 9 & $4,55 \%$ \\
\hline & $21-30$ & 23 & $11,6 \%$ \\
\hline & $31-40$ & 34 & $17,17 \%$ \\
\hline & $41-50$ & 55 & $27,78 \%$ \\
\hline & $51-60$ & 35 & $17,68 \%$ \\
\hline & $61-70$ & 30 & $15,15 \%$ \\
\hline & 71 ou mais & 12 & $6,06 \%$ \\
\hline \multirow{2}{*}{$\begin{array}{l}\text { Morador de } \\
\text { Araraquara: }\end{array}$} & Sim & 169 & $85,35 \%$ \\
\hline & Não & 29 & $14,65 \%$ \\
\hline \multirow[t]{6}{*}{ Bairro: } & $\begin{array}{c}\text { Região Central e Vila } \\
\text { Xavier }\end{array}$ & 7 & $8,28 \%$ \\
\hline & Nordeste & 30 & $17,75 \%$ \\
\hline & Leste & 30 & $17,75 \%$ \\
\hline & Noroeste & 21 & $12,43 \%$ \\
\hline & Demais regiões & 71 & $42,01 \%$ \\
\hline & Assentamento & 3 & $1,78 \%$ \\
\hline \multirow{6}{*}{ Cidade de Origem: } & São Carlos & 10 & $34,48 \%$ \\
\hline & Américo Brasiliense & 6 & $20,69 \%$ \\
\hline & Rincão & 3 & $10,34 \%$ \\
\hline & Descalvado & 2 & $6,90 \%$ \\
\hline & Matão & 3 & $10,34 \%$ \\
\hline & Outras & 5 & $17,24 \%$ \\
\hline \multirow{6}{*}{ Profissão: } & Nivel superior & 2 & $1,01 \%$ \\
\hline & Doméstica & 17 & $8,59 \%$ \\
\hline & Do lar & 51 & $25,76 \%$ \\
\hline & Aposentado & 37 & $18,69 \%$ \\
\hline & Outros & 83 & $41,92 \%$ \\
\hline & Estudantes & 8 & $4,04 \%$ \\
\hline \multirow[t]{2}{*}{ Está empregado: } & Sim & 83 & $41,92 \%$ \\
\hline & Não & 115 & $58,08 \%$ \\
\hline
\end{tabular}

Fonte: Dados de pesquisa.

No que diz respeito ao gênero, observou-se prevalência do sexo feminino $(65,66 \%)$ em comparação com o masculino $(34,34 \%)$. As faixas etárias predominantes entre os entrevistados foram de 41 a 50 anos de idade $(27,78 \%)$ e de 51 a 60 $(17,68 \%)$, e os idosos (61 ou mais) representaram 
$21,21 \%$ da amostra.

Notou-se que $85,35 \%$ dos indivíduos que procuraram por atendimento odontológico eram moradores locais (município de Araraquara) e os demais se originaram de cidades da região como São Carlos (10), Rincão (3), Américo Brasiliense (6), Matão (3), Descalvado (2) e Outras (5). Os bairros mais comuns citados na amostra como local onde residem foram os da região Nordeste e Leste, representado 17,75\% dos participantes para casa uma delas.

Ao serem questionados sobre a profissão que atuam, verificou-se que a amostra foi composta em maior número por pessoas que cuidam "do lar" $(25,76 \%)$ ou por aposentados $(18,69 \%)$. Profissões de nível superior foram as menos citadas $(1,01 \%)$, enquanto outras profissões representaram $41,92 \%$. Os resultados mostraram que $58,08 \%$ da população estudada não se encontrava inserida no mercado de trabalho durante a pesquisa.

O grau de instrução dos pacientes estudados está descrito em forma de gráfico (Figura 1). Nele gráfico é possível observar que a maioria dos entrevistados possui até a $4 .{ }^{a}$ série e apenas uma $3,54 \%$ dos mesmos possuem nível superior.

\section{Grau de escolaridade}

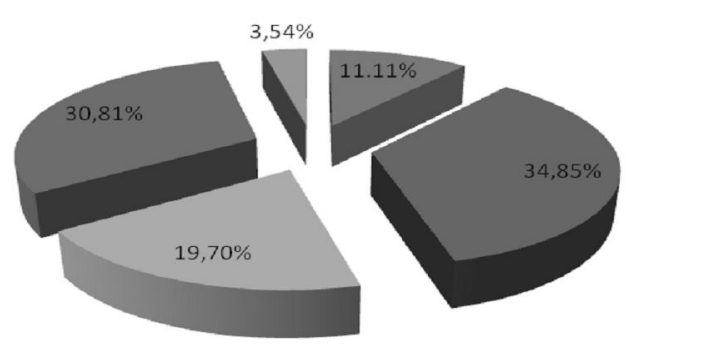

$\begin{array}{ll} & \text { Analfabeto até a } 3^{\mathrm{a}} \text { série } \\ \text { - } & \text { Até a } 4^{\mathrm{a}} \text { série } \\ \text { - } \quad \text { Mundamental completo completo } \\ \text { Superior completo }\end{array}$

Figura 1 - Distribuição de frequência absoluta (n) e relativa (\%) dos aspectos sociais e biodemográficos dos pacientes que buscaram atendimentos na Clínica de Odontologia da UNIARA-Araraquara, 2011-2012.

Fonte: Dados de pesquisa.

Já a análise do perfil econômico dos pacientes entrevistados está apresentada na Tabela 2. 
Tabela 2 - Distribuição de frequência absoluta (n) e relativa (\%) dos aspectos econômicos dos pacientes que buscaram atendimentos na Clínica de Odontologia da UNIARA - Araraquara, 2011-2012.

\begin{tabular}{|c|c|c|c|}
\hline Perguntas & Respostas & $\begin{array}{l}\text { Número de } \\
\text { Pessoas }\end{array}$ & Porcentagem \\
\hline \multirow[t]{4}{*}{ Tipo de moradia: } & Alugada & 41 & $20,71 \%$ \\
\hline & Própria & 148 & $74,75 \%$ \\
\hline & Imóvel cedido & 8 & $4,04 \%$ \\
\hline & Asilo & 1 & $0,51 \%$ \\
\hline \multirow[t]{3}{*}{ Membros familiares: } & 1 & 12 & $6,06 \%$ \\
\hline & $2 a 4$ & 149 & $75,20 \%$ \\
\hline & 5 ou mais & 37 & $18,70 \%$ \\
\hline \multirow[t]{4}{*}{ Renda familiar: } & Até 1 salário & 26 & $13,13 \%$ \\
\hline & Até 3 salários & 96 & $48,49 \%$ \\
\hline & Até 5 salários & 54 & $27,27 \%$ \\
\hline & Mais que 5 salários & 22 & $11,11 \%$ \\
\hline \multirow{8}{*}{ Classe social: } & $A 1$ & 0 & $0 \%$ \\
\hline & $A 2$ & 0 & $0 \%$ \\
\hline & $B 1$ & 5 & $2,53 \%$ \\
\hline & $B 2$ & 51 & $25,76 \%$ \\
\hline & $C 1$ & 76 & $38,38 \%$ \\
\hline & $C 2$ & 38 & $19,19 \%$ \\
\hline & $D$ & 24 & $12,12 \%$ \\
\hline & $E$ & 4 & $2,02 \%$ \\
\hline \multirow[t]{2}{*}{ Computador: } & Sim & 114 & $57,58 \%$ \\
\hline & Não & 84 & $42,42 \%$ \\
\hline \multirow[t]{2}{*}{ Acessa a internet: } & Sim & 78 & $39,39 \%$ \\
\hline & Não & 120 & $60,61 \%$ \\
\hline \multirow[t]{2}{*}{ Atividade de lazer: } & Sim & 67 & $33,84 \%$ \\
\hline & $N \tilde{a} o$ & 131 & $66,16 \%$ \\
\hline \multirow{4}{*}{ Qual atividade de lazer: } & Esporte & 33 & $49,25 \%$ \\
\hline & Cultura & 4 & $5,97 \%$ \\
\hline & Diversão & 7 & $10,45 \%$ \\
\hline & Outros & 23 & $34,33 \%$ \\
\hline
\end{tabular}

Fonte: Dados de pesquisa.

Observa-se que $74,75 \%$ da população estudada possui moradia própria. A maioria dos entrevistados $(75,20 \%)$ vive com 2 a 4 membros na mesma residência, com renda familiar variando entre 3 a 5 salários $(48,49 \%$ e 27,27\%), respectivamente, sendo poucos $(11,11 \%)$ os indivíduos com renda maior (5 salários ou mais).

Com base nas respostas obtidas, notou-se que 
nenhum participante da pesquisa pertencia às classes A1 eA2, sendo as classes econômicas mais prevalentes a B2 $(25,76 \%)$, C1 $(38,38 \%)$ e C2 $(19,19 \%)$.
Quanto ao perfil da necessidade de atendimento e os conhecimentos sobre a Clínica de Odontologia da UNIARA, os resultados são encontrados na Tabela 3.

Tabela 3 - Distribuição de frequência absoluta (n) e relativa (\%) do perfil da necessidade atendimento odontológica dos pacientes que buscaram atendimento na Clínica de Odontologia da UNIARAAraraquara, 2011-2012.

\begin{tabular}{|c|c|c|c|}
\hline Perguntas & Respostas & $\begin{array}{l}\text { Número } \\
\text { de } \\
\text { Pessoas }\end{array}$ & Porcentagem \\
\hline \multirow{4}{*}{$\begin{array}{l}\text { Meio de transporte utilizado } \\
\text { para chegar à clínica }\end{array}$} & Coletivo & 100 & $50,51 \%$ \\
\hline & Particular & 76 & $38,38 \%$ \\
\hline & Ambos & 2 & $1,01 \%$ \\
\hline & Nenhum & 20 & $10,10 \%$ \\
\hline \multirow{2}{*}{$\begin{array}{l}\text { Tem conhecimento do } \\
\text { atendimento odontológico } \\
\text { oferecido na rede pública: }\end{array}$} & Sim & 146 & $73,74 \%$ \\
\hline & Não & 52 & $26,26 \%$ \\
\hline \multirow{2}{*}{$\begin{array}{l}\text { Antes do atendimento na } \\
\text { UNIARA passou por dentista } \\
\text { público ou privado: }\end{array}$} & Sim & 127 & $64,14 \%$ \\
\hline & Não & 71 & $35,86 \%$ \\
\hline \multirow{3}{*}{$\begin{array}{l}\text { Se sim, como foi o } \\
\text { atendimento prestado: }\end{array}$} & Ótimo & 28 & $22,05 \%$ \\
\hline & Bom & 71 & $55,91 \%$ \\
\hline & Ruim & 28 & $22,05 \%$ \\
\hline \multirow{5}{*}{$\begin{array}{l}\text { Como obteve conhecimento } \\
\text { da clínica da UNIARA: }\end{array}$} & $\begin{array}{l}\text { Dentista } \\
\text { particular }\end{array}$ & 9 & $4,55 \%$ \\
\hline & $\begin{array}{l}\text { Dentista da rede } \\
\text { pública }\end{array}$ & 55 & $27,78 \%$ \\
\hline & Amigos & 63 & $31,82 \%$ \\
\hline & Por conta própria & 21 & $10,61 \%$ \\
\hline & Outros & 50 & $25,25 \%$ \\
\hline \multirow{8}{*}{ Qual o motivo da consulta: } & $\begin{array}{l}\text { Indicação } \\
\text { dentista } \\
\text { particular }\end{array}$ & 4 & $2,02 \%$ \\
\hline & $\begin{array}{l}\text { Indicação } \\
\text { dentista rede } \\
\text { pública }\end{array}$ & 34 & $17,17 \%$ \\
\hline & $\begin{array}{l}\text { Indicação por } \\
\text { amigo }\end{array}$ & 2 & $1,01 \%$ \\
\hline & Dor & 71 & $35,86 \%$ \\
\hline & Incomodo & 31 & $15,66 \%$ \\
\hline & Rotina & 20 & $10,10 \%$ \\
\hline & Baixo custo & 17 & $8,59 \%$ \\
\hline & Outros & 19 & $9,60 \%$ \\
\hline
\end{tabular}

Fonte: Dados de pesquisa. 
Ao serem abordados sobre o tipo de transporte utilizado para o deslocamento até a clínica, 50,51\% afirmaram usar o transporte público coletivo.

Sobre o atendimento odontológico da rede pública, $73,74 \%$ disseram ter conhecimento do serviço prestado e $61,14 \%$ já haviam sido atendidos por esse mesmo serviço ou em serviço particular antes de recorrerem à UNIARA. Alguns entrevistados $(27,78 \%)$ relataram ter obtido a informação da prestação de serviço odontológico pela IES por meio do próprio cirurgião dentista da rede pública.

No que diz respeito ao motivo da consulta, a maior procura ocorreu devido à presença de dor $(35,86 \%)$, seguida de incômodo na cavidade bucal $(15,66 \%)$. Somente $10,10 \%$ da amostra consideraram a necessidade de atendimento de rotina e $8,59 \%$ relacionaram a procura pelo serviço em decorrência do baixo ou nenhum custo dos procedimentos.

\section{Discussão}

Na última década, o Brasil tem passado por mudanças socioeconômicas que vêm transformando a sociedade brasileira e possibilitando, assim, uma melhor ascensão social. Um reflexo disso é o surgimento da nova classe $\mathrm{C}$, formada por pessoas com um maior poder aquisitivo, que estão comprando mais, viajando mais, se preocupando com a saúde e dando mais importância para aparência, a qual abre portas para um melhor emprego e possibilidade de melhoria de vida. Nesse sentido, para a boa apresentação pessoal, um belo sorriso é de fundamental importância. Por essa razão, nos últimos anos vem aumentando consideravelmente o número de pessoas que procuram atendimento em Centros Universitários Odontológicos, em Clínicas e em Centros de Especializações. Considerando-se que ainda são poucos os estudos relacionados a essa nova classe $C$, é relevante conhecer quem são os pacientes que procuram atendimento nas Clínicas de Odontologia das Faculdades brasileiras. Segundo Brandini et al. (2008), conhecer o padrão socioeconômico de usuários de serviços públicos é de extrema importância no processo de planejamento das atividades a serem realizadas nas Clínicas de
Instituições de Ensino Superior - IES, bem como é determinante na construção do plano de tratamento a ser proposto para cada paciente. Portanto, reconhecer os perfis socioeconômico, cultural e demográfico dos pacientes atendidos nas Clínicas de Odontologia da UNIARA possibilita corrigir possíveis falhas na logística do atendimento, na diminuição das faltas às consultas agendadas, assim como no planejamento das atividades clínicas desenvolvidas no decorrer do ano.

Dessa forma, o presente trabalho buscou conhecer o perfil dos pacientes atendidos pelas Clínicas de Odontologia do Centro Universitário de AraraquaraUNIARA. Como resultado, foi possível observar que a maioria dos usuários $(65,66 \%)$ pertence ao gênero feminino (Tabela 1). Emestudos anteriores sobre análise do perfil socioeconômico de pacientes de Clínicas Universitárias Odontológicas, o gênero prevalente também foi o feminino, variando entre $70,7 \%, 65 \%$ e 56,60. Essa maior demanda da mulher ao serviço de saúde pode estar associada à questão cultural ou social, em que a mulher normalmente é responsável por acompanhar o filho e os idosos ao médico, além de realizar o pré-natal, tornando-a naturalmente mais disposta a frequentar os serviços de saúde. Além disso, é provável também que o maior número de prontuários de mulheres esteja relacionado somente a maior incidência do gênero feminino sobre o masculino na população da região estudada. Em estudo realizado por Pimentel et al. (2008) em relação ao perfil demográfico de pacientes com dor orofacial, foi verificada a prevalência $(84 \%)$ de mulheres nos atendimentos realizados na Faculdade de Odontologia de Pernambuco.

Com relação à faixa etária dos entrevistados do estudo em questão, grande parcela dos indivíduos $(27,78 \%)$, independente do gênero, encontrava-se na faixa etária de 41 a 50 anos (Tabela 1). Já em um estudo semelhante realizado por Watanabe et al. (1997) foi verificada que a maior parte dos indivíduos entrevistados se encontrava na faixa etária entre 18 a 50 anos $(56,4 \%)$, dado muito próximo ao obtido neste estudo, em que a soma das porcentagens dos entrevistados na faixa etária de 21 a 50 anos é de 
$56,55 \%$ (Tabela 1). Entretanto, no estudo de Moreira et al. (2006) foi relatado que as faixas etárias dos pacientes atendidos nas Clínicas Odontológicas na Universidade de Marilia-UNIMAR eram diversificadas, assim como a cor e o estado civil.

Em se tratando do grau de escolaridade, 34,5\% dos entrevistados possuem até a 4 a série do ensino fundamental completo e $30,84 \%$, o ensino médio completo. Além disso, essa avaliação mostra também que pessoas com um maior grau de escolaridade foram as que menos procuraram atendimento Odontológico na UNIARA $(3,53 \%)$ (Tabela 1). Carvalhais et al. (2001) também observaram que a maior parcela dos entrevistados possuía primário completo, assim como verificado neste trabalho. Esse dado pode ser explicado pelo fato de que os indivíduos com maior grau de escolaridade, geralmente, possuem melhor condição financeira e não se submetem a procedimentos realizados por alunos em fase de aprendizagem.

$\mathrm{O}$ estudo mostrou que a maioria dos atendimentos ocorre para pacientes deste município $(85,35 \%)$, sendo a maior concentração entre indivíduos de bairros mais afastados e carentes (Regiões Leste, Noroeste e alguns bairros da região Nordeste), que juntos correspondem a 47,93\% da procura. De acordo com Campos et al. (2012), em estudo comparativo entre municípios de melhor e pior indicador socioeconômico, foi observado que a cobertura de Equipe de Saúde da Família e o número de visitas domiciliares foram maiores nos municípios com piores indicadores socioeconômicos, já que a proposta da Estratégia de Saúde da Família - ESF é atingir populações com menores recursos financeiros. No entanto, embora Araraquara apresente um índice de IDH elevado $(0,83)$, a cobertura da ESF, que ocorre atualmente em 12 bairros, parece ainda ser insuficiente para atender à demanda da população mais carente, o que pode justificar a maior procura por atendimento na IES estudada por este público.

Outro resultado obtido neste trabalho foi a identificação da cidade de origem dos frequentadores da Clínica de Odontologia da UNIARA. Notou-se que $14,65 \%$ dos entrevistados são de cidades da região de Araraquara (Tabela 1), comprovando que as faculdades têm um papel importante na promoção de saúde, com um atendimento gratuito, que abrange não somente a cidade a qual está localizada, mas também toda a região. Em 2011, Sanchez e Drumond também relataram que uma grande parcela $(46,3 \%)$ dos pacientes que compareceram ao atendimento de urgência da Universidade Federal de Minas Gerais era de locais distantes, incluindo outros municípios, o que deixou claro para os autores que as ações ou programas existentes não são suficientes para atender à demanda populacional por essa modalidade de atendimento. Outro estudo, realizado por Batista et al. (2010), em que graduandos de Odontologia, ao participar de estágio em Unidades de Saúde da Família de Piracicaba, relataram a falta de recursos na atenção primária como pontos negativos do atendimento da rede pública, como exemplos: aparelhos de $\mathrm{RX}$, tratamentos endodônticos e próteses. Isso reforça a questão de haver maior procura do atendimento nas universidades, uma vez que outros serviços de saúde não oferecem todos os diferentes procedimentos odontológicos.

Em relação à questão do desemprego, pode-se observar que $58,08 \%$ dos entrevistados não trabalhavam no período em que participaram da pesquisa na Clínica Odontológica da UNIARA. Esse resultado está de acordo com o encontrado por Nassri et al. (2009), em que $50 \%$ dos entrevistados não trabalhavam no período em que procuraram o tratamento na Clínica Odontológica na Universidade estudada pelos autores. No que diz respeito à profissão, $25,76 \%$ dos pacientes entrevistados neste estudo eram do lar (Tabela 1), ou seja, sem remuneração alguma. Em contrapartida, o estudo de Cidral et al. (2004) mostrou que a maioria $(52,09 \%)$ da população atendida pela Clínica de Ensino Odontológico da Universidade do Vale do Paraíba - UNIVAP se encontra no mercado de trabalho.

Como no estudo realizado anteriormente por D'Ávila et al. (2010), em que 75,5\% dos frequentadores das Clínicas de Odontologia da Universidade Estadual da Paraíba possuíam casa 
própria, o presente trabalho mostrou que 74,75\% dos entrevistados na UNIARA possuem moradia própria e 75,20\% moram com 4 até pessoas na mesma casa (Tabela 2). Pode-se observar também que 48,49\% dos pacientes entrevistados da UNIARA possui uma renda mensal de até 3 salários mínimos (Tabela 2), diferentemente da renda mensal descrita por Cidral et al. (2004), onde 52,47\% dos entrevistados possuíam a faixa de um salário mínimo. No estudo de Victor $e t$ al. (2009), a maior concentração dos pacientes foi daqueles com renda máxima de 1,5 salário mínimo (81,3\%); já Picapedra (2009) verificou que $38 \%$ das famílias dos pacientes que participaram do estudo na Facultad de Odontologia de La Universidad de La Repúplica Del Uruguay - FO-UDELAR tinham uma renda mensal entre 5 a 10 salários da moeda local. Um fato notado durante a entrevista dos participantes da presente pesquisa com relação a esse tópico é que foi observado um desconforto pelos pacientes que procuram a UNIARA quando questionados sobre a sua renda familiar, mostrando que, no entendimento dessas pessoas, o serviço prestado pelos Cursos de Odontologia seria destinado somente para indivíduos sem condições financeiras para receber atendimento odontológico particular. Porém, essa não é a realidade do Curso de Odontologia do Centro Universitário de Araraquara, uma vez que o atendimento é destinado a toda população, independentemente da condição social ou financeira.

Já a análise da classe social realizada neste estudo dos frequentadores da UNIARA mostrou que o maior número dos entrevistados $(38,38 \%)$ pertencia à classe C1 (Tabela 2), a qual é determinada pela renda familiar de até $\mathrm{R} \$ 1.541,00$. Essa classificação é efetuada com base no Critério de Classificação Econômica Brasil, que é obtida por meio de um sistema de pontos que agrupa as famílias em classes sociais (A1, A2, B1, B2, C1, C2, D e E). Neste estudo não foram encontrados indivíduos pertencentes às classes sociais A1 e A2 (Tabela 2). Esse resultado foi semelhante ao encontrado por Carvalhais et al. (2001), em que foi observado que as classes sociais mais representadas nas Clínicas de Endodontia da Universidade Federal de Minas Gerais - FO/UFMG correspondiam à classe C e à classe $\mathrm{D}($ com $43,01 \%$ e 38,7\%, respectivamente); e, assim como neste trabalho, não foi atendido nenhum paciente das classes A1 e A2. Watanabe $e t$ al. (1997) afirmaram que, apesar de a maior parte dos pacientes que responderam a um questionário sobre aspectos socioeconômicos das Clínicas Odontológicas da Universidade de São Paulo de Ribeirão Preto, possuir uma condição socioeconômica considerada menos privilegiada, ou seja, com baixa renda familiar per capita mensal e baixo nível de escolaridade, observou-se que o serviço também foi procurado por pessoas com condições de vida mais favorecidas.

Já em relação ao meio de transporte usado pelos pacientes para frequentar as Clínicas Odontológicas da UNIARA, 50,51\% utilizava o transporte coletivo (Tabela 3), o qual se refere ao transporte público municipal e ao transporte cedido pelas prefeituras. Além disso, neste estudo também foi analisado se os pacientes entrevistados procuraram atendimento odontológico público ou privado antes de procurar o atendimento Odontológico na UNIARA. Sendo assim, $64,14 \%$ relataram que já haviam sim passado por atendimento e, destes, $55,91 \%$ consideraram o atendimento como bom (Tabela 3 ).

Em relação à obtenção de informações sobre o atendimento prestado pela Clínica Odontológica da UNIARA, 31,82\% dos entrevistados relataram que conheceram tal serviço por intermédio de amigos, seguido de $27,78 \%$ por indicação de dentista da rede pública (Tabela 3), de acordo com relato de alguns pacientes que procuraramatendimento na Faculdade de Odontologia da UNESP - Campus Araraquara, o fato de existir fila de espera e tempo indeterminado para agendamento do início do tratamento, ou ainda, por não haver atendimento de determinadas disciplinas no momento da procura, os mesmos foram orientados a buscar atendimento no Centro Universitário de Araraquara - UNIARA. Já Borghi et al. (2008) verificaram que a informação do atendimento odontológico na Clínica Integrada da UNESP de Araçatuba atinge aos pacientes por fontes espontâneas e variadas. 
Finalmente, foi constatado que a maior parte dos pacientes entrevistados $(35,86 \%)$ neste trabalho relatou ter procurado atendimento na Clínica de Odontologia na UNIARA pelo motivo de dor (Tabela 3). Porém, em diferentes estudos foram observados diferentes motivos para os pacientes terem procurado atendimento odontológico, como rotina, emergência, procedimentos de média complexidade, reabilitação ou serviços de especialidade.

Dessa forma, o presente estudo forneceu conhecimento sobre o perfil social, demográfico e econômico dos pacientes atendidos na Clínica de Odontologia do Centro Universitário de Araraquara UNIARA, o qual poderá ser utilizado para elevar a qualidade de planejamento dos atendimentos clínicos oferecidos à população que anualmente recorre ao Curso de Odontologia da UNIARA.

\section{Conclusão}

Frente ao exposto, concluiu-se que o perfil da população estudada foi de:

- maioria do gênero feminino;

- moradores do município de Araraquara/SP;

- pertencentes à classe $C$;

- procuraram atendimento odontológico na Instituição estudada pelo motivo de dor ou incômodo.

\section{REFERÊNCIAS}

ABREU, M.H.N.G.; OLIVEIRA, R.FR.

Características sociodemográficas dos usuários das Clínicas Integradas I e II do Curso de Odontologia da Universidade de Montes Claros, Minas Gerais, Brasil. Unimontes Científica, v.4, n.2, p.1-12, 2002.

\footnotetext{
ALMEIDA, G.L.; GARCIA, L.F.R.; ALMEIDA, T.L.; BITTAR, T.O.; PEREIRA, A.C. Estudo do perfil sócio-econômico dos pacientes e os motivos que os levaram a faltar em consultas odontológicas na estratégia de saúde da família em uma distrital de Ribeirão Preto/SP. Cienc. Odontol. Bras., v.12, n.1, p.77-86, 2009.
}

BALDANI, M.H.; VASCONCELOS, A.G.G.; ANTUNES, J.L.F. Associação do índice CPO-D com indicadores sócio-econômicos e de provisão de serviços odontológicos no Estado do Paraná, Brasil.

Cad. Saúde Pública, v.20, n.1, p.143-152, 2004.

BATISTA, M.J.; GIBILINI, C.; KOBAYASHI, H.M.; FERREIRA, L.L.; GONÇALO, C.S.; SOUSA, M.L.R. Relato de experiência da interação entre Universidade, comunidade e Unidade de saúde da família em Piracicaba, SP, Brasil. Arq. Odontol., v.46, n.3, p.144-151, 2010.

BERTOLI, L.C.F.; FERRONATO, T. Perfil dos pacientes com necessidades especiais atendidos na Faculdade de Odontologia da Universidade Federal do Rio Grande do Sul. [TCC]. Porto Alegre: UFRGS; 2009.

BORGHI, W.M.M.C.; SUNDFELD, M.L.M.M.; SALIBA, N.A.; MOIMAZ, S.A.S.; POI, W.R. Razões que influenciam o paciente a buscar atendimento odontológico na Clínica Integrada. Pesq Bras Odont., v.8, n.3, p.347-352, 2008.

BRANDINI, D.A.; POI, W.R.; MELLO, M.L.M.; MACEDO, A.P.A.; PANZARINI, S.R.; PEDRINI, D.; CASTRO, R.D. Caracterização social dos pacientes atendidos na disciplina de Clínica Integrada da Faculdade de Odontologia de Araçatuba, UNESP. Pesq Bras Odontoped Clin Integr., v.8, n.2, p.245-250, 2008.

CAMPOS, A.C.V.; BORGES, C.M.; LELES, C.R.; VARGAS, A.M.D.; FERREIRA, E.F. Indicadores socioeconômicos e de saúde da Atenção Básica nos municípios da região metropolitana de Belo Horizonte. Arq. Odontol., v.48, n.1, p.6-12, 2012.

CARVALHAIS, H.P.M.; MALTOS, K.L.M.; FARIA, R.A.; MALTOS, S.M.M.; CALVALCANTI, A.L.N.; OLIVEIRA, A.P.L. 
Levantamento das classes econômicas dos pacientes atendidos nas Clínicas de Endodontia da FO/ UFMG. Arq Odontol., v.37, n.1, p.45-51, 2001.

CIDRAL, V.; MARTINS, A.; CARVALHO, F.; NASCIMENTO, P.; NUNES, P.; GUELFI, D.C. Levantamento do perfil socioeconômico da população atendida pela Policlínica de Ensino Odontológico da Faculdade de Ciências da Saúde. In: ENCONTRO LATINO AMERICANO DE INICIAÇÃO CIENTÍFICA, 9; ENCONTRO LATINO AMERICANO DE PÓSGRADUAÇÃO, 5 - Universidade do Vale do Paraíba, 2005. p.706-8.

CRITÉRIO de Classificação Econômica Brasil CCEB 2011 base LES 2009; disponível em http:// www.abep.org/novo/Content.aspx?ContentID=301, acesso em: 18 de maio 2011.

D'ÁVILA, S.; SOUZA, L.M.M.; CAVALCANTI, G.M.S.; LUCAS, R.S.C.C.; GARCIA, A.F.G.; CAVALCANTI, A.L. Nível de satisfação e condição socioeconômica dos usuários das Clínicas de Odontologia da Universidade Estadual da Paraíba. Rev. Bras. Pesq. em Saúde., v.12, n.2, p.39-45, 2010.

GONÇALVES, E.R.; VERDI, M.I.M. Os problemas éticos no atendimento a pacientes na Clínica Odontológica de Ensino. Cienc. Saúde Colet., v.12, n.3, p.755-764, 2007.

MOREIRA, A.C.; MILANEZI, L.A.; OKOMOTO, T.; OKOMOTO, R. Perfil de pacientes submetidos a procedimentos odontológicos na Faculdade de Ciências da Saúde da Universidade de Marília (UNIMAR)-SP, em 2003. Rev. Odontol. Araçatuba., v.27, n.2, p.136141, 2006.

MOREIRA, R.S.; NICO, L.S.; SOUSA, M.L.R. Fatores associados à necessidade subjetiva de tratamento odontológico em idosos brasileiros. Cad. Saúde Pública., v.25, n,12, p.2661-2671, 2009.

NAKAMURA, C.C.; GONÇALVES, D.R.; CASTRO, R.F.M.; CLOSS, P.S. Perfil dos pacientes atendidos na Clínica Odontológica da Faculdade São Lucas, Porto Velho-RO. Saber Cient Odontol., v.1, n.1, p.42-52, 2010.

NASSRI, M.R.G.; SILVA, A.S.; YOSHIDA, A.T. Levantamento do perfil socioeconômico de pacientes atendidos na Clínica Odontológica da Universidade de Mogi das Cruzes e do tratamento ao qual foram submetidos: clínica endodôntica.

RSBO, v.6, n.3, p.272-278, 2009.

PICAPEDRA, A. Determinação do perfil socioeconômico e cultural dos pacientes atendidos na Faculdade de Odontologia de La Universidad de La República Del Uruguay (FO-UDELAR), por meio dos prontuários odontológicos no período de junho de 2006 a agosto de 2007. [Monografia]. Piracicaba: Unicamp; 2009.

PIMENTEL, P.H.W.G.; COELHO JR, L.G.T.M.; CALDAS JR, A.F.; KOSMINSKY, M.; AROUCHA, J.M.C.N.L. Perfil demográfico dos pacientes atendidos no Centro de Controle da Dor Orofacial da Faculdade de Odontologia de Pernambuco. Rev Traumatol. Buco-Maxilo-Fac., v. 8, n.2, p.69-76, 2008.

SAMPAIO, E.F.; CÉSAR, F.N.; MARTINS, M.G.A. Perfil odontológico dos pacientes portadores de necessidades especiais atendidos no Instituto de Previdência do Estado do Ceará.

RBPS., v.17, n.3, p.127-134, 2004.

SANCHEZ, H.F.; DRUMOND, M.M.

Atendimento de urgências em uma Faculdade de Odontologia de Minas Gerais: perfil do paciente e resolutividade. Rev. Gaúcha Odontol., v.59, n.1, p.79-86, 2011. 
TIEDMANN, C.R.; LINHARES, E.; SILVEIRA， Saúde da Família. Acta Paul. Enferm., v.22, n.1, J.L.G.C. Clínica Integrada Odontológica: perfile p.49-54, 2009. expectativas dos usuários e alunos. Pesq. Bras.

Odontoped. Clin. Integr., v.5, n.1, p.53-58, 2005. VICTOR, J.F.; XIMENES, L.B.; ALMEIDA, P.C.; VASCONCELOS, F.F. Perfil sociodemográfico e clínico de idosos atendidos em Unidade Básica de
WATANABE, M.G.C.; AGOSTINHO, A.M.; MOREIRA, A. Aspectos socioeconômicos dos pacientes atendidos nas Clínicas da Faculdade de Odontologia de Ribeirão Preto - USP. Rev. Odontol. Univ. São Paulo, v.11, n.2, p.147-151, 1997. 